\title{
Studies of synoptic solar activity using Kodaikanal Ca K data
}

\author{
K. P. Raju \\ Indian Institute of Astrophysics, \\ Bangalore 560034, India \\ email: kpr@iiap.res.in
}

\begin{abstract}
The chromospheric network, the bright emission network seen in the chromospheric lines such as $\mathrm{Ca}$ II $\mathrm{K}$ and $\mathrm{H}_{\alpha}$, outline the supergranulation cells. The $\mathrm{Ca}$ images are dominated by the chromospheric network and plages which are good indicators of solar activity. Further, the Ca line is a good proxy to the UV irradiance which is particularly useful in the pre-satellite era where UV measurements are not available. The Ca spectroheliograms of the Sun from Kodaikanal have a data span of about 100 years and covers over 9 solar cycles. The archival data is now available in the digitized form. Programs have been developed to obtain the activity indices and the length scales of the chromospheric network from the data. The preliminary results from the analysis are reported here. It is shown that the Ca II K intensity and the network boundary width are dependent on the solar cycle.
\end{abstract}

Keywords. Sun: activity, Sun: chromosphere, Sun: magnetic fields

\section{Introduction}

The observations at Kodaikanal Solar Observatory date back to the year 1904 (Bappu 1967). The archival data have a span of about 100 years which covers over 9 solar cycles. The database consists of

- Ca II K spectroheliograms at $3934 \AA$

- $\mathrm{H}_{\alpha}$ spectroheliograms at $6563 \AA$

- White light images

The data is now available in the digitized format and can be used for studying the synoptic variations in the Sun (Priyal et al. 2014).

The Ca II K line at $3934 \AA$ is a strong chromospheric line. The Ca images are dominated by chromospheric network and plages (Raju \& Singh 2014). It is well known that the chromospheric network outlines supergranular cells which play an important role in maintaining solar cycle through magnetic flux dispersal along its boundaries. Therefore the Ca line is a good tracer of solar activity.

Solar irradiance variation is about $0.1 \%$ over solar cycle. However, the variation of UV irradiance is much higher, about $10 \%$ and it plays an important role in Earth's climate. The Ca line is a proxy to UV irradiance which is particularly useful in the pre-satellite era.

The length scales of supergranulation network can be obtained through the autocorrelation method of Ca II K images. The half-width of the autocorrelation function gives the width of the network boundary (Patsourakos et al. 1999). The solar cycle variation of the transition region EUV network boundaries has been reported recently by Raju (2016). In the present work, we examine the synoptic variation of chromospheric network in the Ca II K line from the Kodaikanal archival data. The recent IRIS observations have reported 


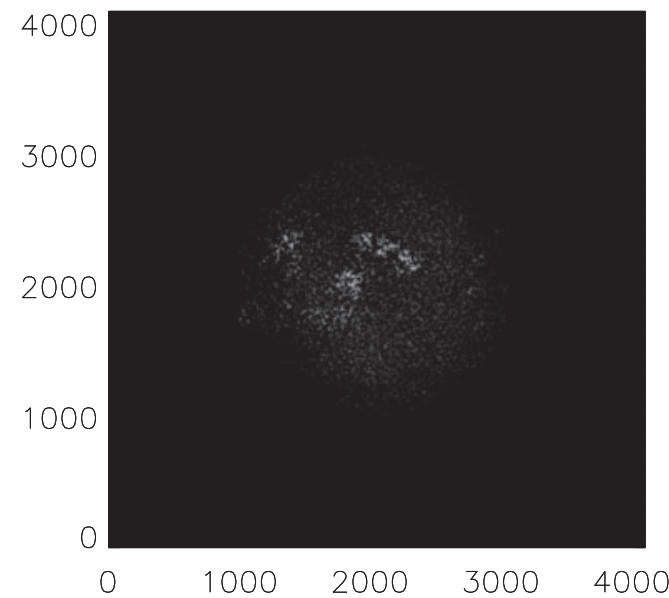

Figure 1. Ca II K Spectroheliogram from Kodaikanal.

the presence of jets and loops in the network boundaries (Tian et al. 2014). Hence the variations of network length scales are important in the mass and energy budget of the solar atmosphere.

\section{Data and Analysis}

The Kodiakanal Ca II K spectroheliograms have a spatial resolution of about 2 arc sec. The data was recently digitized using a $4 \mathrm{~K} \times 4 \mathrm{~K}$ CCD with a pixel resolution of 0.86 arc sec and then calibrated to intensity units (Priyal et al. 2013). The spectroheliograms cover a period from 1907 to 2008. An example of the calibrated image is shown in Figure 1.

During the analysis, a strip of width $480 \operatorname{arc~sec~} \mathrm{x} 45$ arc sec was selected from the solar centre. The strip contains about 12 supergranules which will give the statistical behaviour of the cells. First the mean intensity of the strip was obtained. Any possible large-scale trend was then removed from the strip by fitting a polynomial fit and subtracting it out. The two-dimensional autocorrelation function as a function of lag was obtained through an IDL routine. The width of the network boundary as a function of time was then obtained from the spectroheliograms.

\section{Results}

The results are plotted in Figure 2. The panels give the sunspot number, Ca II K intensity and the network boundary width against time. It can easily be seen that both the intensity and the boundary width have a rough positive correlation with the sunspot number. Since the network is formed due to the concentration of magnetic flux by solar convection, the correlation is rather expected. As the cycle rises, more and more flux reaches the network boundary and adds to its width. The result agrees well with that from the transition region network (Raju (2016)). The implications of the result to the mass and energy budget of the solar atmosphere need to be examined through modelling. As a future work, we plan to study the variation of these parameters at different solar latitudes which may give insights on surface flows and flux transport. 


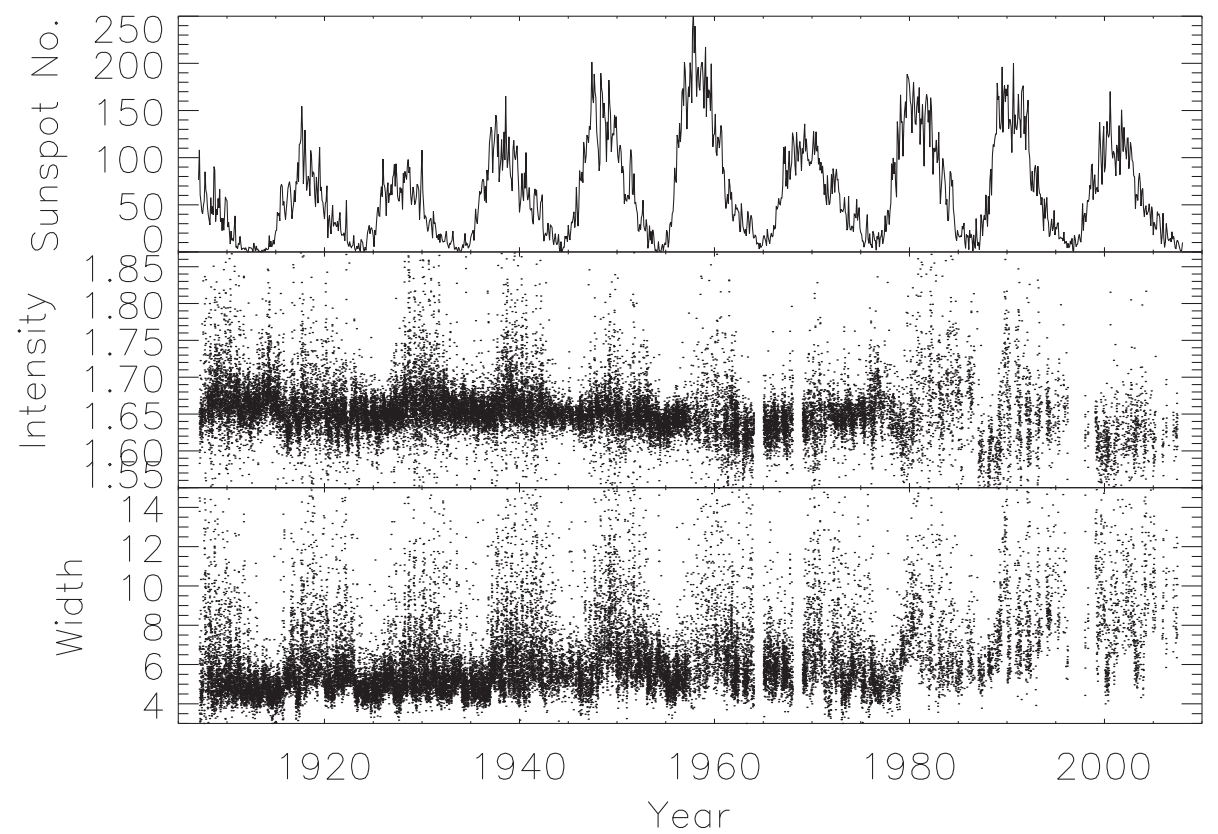

Figure 2. Temporal variation of sunspot number, Ca II K intensity and network boundary width.

\section{References}

Bappu, M. K. V. 1967, Solar Phys., 1, 151

Patsourakos, S., Vial, J.-C., Gabriel, A. H., \& Bellamine, N. 1999, ApJ, 522, 540

Priyal, M., Singh, J., Ravindra, B., Priya, T. G., \& Amareswari, K. 2014, Solar Phys., 289, 137 Raju, K. P. 2016, Solar Phys., 291, 3519

Raju, K. P. \& Singh, J. 2014, Research in Astronomy and Astrophysics, 14, 229

Tian, H., DeLuca, E. E., Cranmer, S. R., De Pontieu, B., Peter, H., Martnez-Sykora, J., et al. 2014, Science, 346, 1255711 